\title{
Dispenser printed capacitive proximity sensor on fabric for applications in the creative industries
}

\author{
Yang Wei, Russel Torah, Yi Li and John Tudor \\ Electronics and Computer Science, \\ University of Southampton, \\ Southampton, \\ SO17 1BJ \\ +44(0) 2380592126 \\ Email: y.wei@soton.ac.uk
}

\begin{abstract}
:
This paper reports a planar capacitive proximity sensor fully dispenser printed on a standard polyester woven fabric using conductive ink. Dispenser printing is a new digital printing technique offering the advantages of complete geometric design flexibility and the ability to direct write multilayer devices without requiring bespoke tooling. A dispenser printer is also capable of printing a wide range of ink viscosities encompassing those of inkjet and screen printable inks. Previous research has demonstrated the principle of using proximity sensors for human interaction but none of them are fabricated directly on fabric. In this research, the proximity sensor is dispenser printed directly onto the fabric with an optimised loop electrode design which uses $76 \%$ less conductive ink while still offering $90 \%$ of the detection range when compared with a standard filled electrode design. The loop design also has the highest detection coefficient (maximum detection distance versus the conductive area of the sensor) of 0.23 compared with 0.06 and 0.1 for the investigated filled and spiral designs, respectively. In addition, the ratio of the track width to the width of the entire sensor is investigated showing $1 / 16$ as being the most suitable ratio for the proximity sensor printed on fabric. Proximity sensors with loop widths ranging from $10 \mathrm{~mm}$ to $400 \mathrm{~mm}$ are evaluated. The maximum detection distance is $400 \mathrm{~mm}$ when the largest sensor is used and the linearity of the sensing circuit is 0.79 .
\end{abstract}

Keywords: Dispenser printer, proximity sensor, smart fabrics, printed electronics, direct write, creative industries

PACS: 07.07.Df

\section{Introduction}

Smart fabrics are fabrics with integrated electronics which are classified into three categories [1]: passive smart fabrics, active smart fabrics and ultra-smart fabrics. Smart fabrics have been widely researched in healthcare, consumer electronics, fashion and the military, and also have many applications within the creative industries. Many creative applications require the smart fabrics, within an artwork or clothing, to be interactive which can be achieved by means of sensors and actuators. Such interaction with the smart fabric results in a more immersive and memorable experience for the user and can be used in fields as diverse as augmented reality and art therapy.

To integrate the sensing element, miniaturised sensors manufactured by silicon microfabrication techniques are often attached to the fabric. However, since these sensors are rigid, they have the potential to cause discomfort if worn. Furthermore, each sensor must be individually mounted on the fabric which is time consuming and costly. Finally, since such a sensor is inherently small, they may not be suitable for large scale creative applications such as in architecture or interior design.

Weaving, knitting and embroidery of electronically functional yarns can also be used to achieve smart fabrics. However, with weaving and knitting, the primary objective is to achieve the desired fabric structure and so the layout of the functional yarn(s) is constrained to coincide with the fabric`s structural layout. In addition, since a yarn must follow the entire 
length of the fabric, difficulties occur with complex designs, in particular where functionalised yarns need to cross since unwanted connections can occur. Furthermore, weaving and knitting methods may not suit the creative industries since yarns must follow orthogonal directions limiting creative design freedom. Embroidery is also used to create conductive tracks on fabric [2] and has no limitation in yarn direction. It can only embroider one type of yarn at a time but achieving multi-layer, multi-material electrically functional devices is difficult because the embroidery process is very abrasive to the yarns and fabric substrate and can therefore damage the previously embroidered layers resulting in short circuits.

Recent dispenser printing research [3-5] offers an alternative digital smart fabric fabrication technique in which the designs are printed, using electronic inks, directly on the fabric in any geometric layout and only where they are needed without the use of masks, screens or other tooling, thus allowing rapid prototyping, minimising resource usage and maximising fabric breathability. Unlike weaving, knitting and embroidery, dispenser printing provides almost complete design freedom since the printed layers can have any orientation on the fabric without being restricted to following the yarn directions. Further, multilayer devices are simply achieved by printing the devices in a layer-by-layer planar fashion using different electronic inks, as required. Compared with other printing techniques, dispenser printing allows materials with a wider range of viscosities (0.01 to 1,000 Pa.s [6]) to be deposited than screen (3 to $250 \mathrm{~Pa}$.s [7]), inkjet (0.001 to $0.02 \mathrm{~Pa}$.s [8]) and aerosol printing (0.001 to $1 \mathrm{~Pa} . \mathrm{s}$ [9]). The thickness of each deposited layer can be controlled by means of the dispense parameters allowing selection of a thickness to achieve continuous electronic layers in a single pass.

A proximity sensor detects the presence or absence of a nearby object within a specified distance without requiring any physical contact. This object can be either a conductor or nonconductor depending on the sensing mechanism of the proximity sensor. Such a proximity sensor can be integrated with other printed electronic actuators to form an interactive fabric. It is therefore suitable for integrating interactivity within a smart fabric since the proximity of a person or object may be used to trigger an event. Proximity sensing has been used for applications, such as motion detection/control [10], process control [11] and level control [12] Sensing the human body such as the arm using proximity sensors has also been reported in the literature [13].

The capacitive mechanism, which changes the measured capacitance of an electrode due to proximity of a grounded object, can be used to sense the proximity of humans and has been achieved on both rigid and flexible substrates [14-17]. Togura et.al. used a commercial sensing module to interact with a person in a car [14]. Two sensing electrodes of $50 \mathrm{~mm} \times$ $50 \mathrm{~mm}$ and $100 \mathrm{~mm} \times 100 \mathrm{~mm}$ are installed in the instrument panel and the ceiling, respectively, achieving a maximum detection distance of $300 \mathrm{~mm}$. Lee et.al. reported a capacitive proximity sensor made of PDMS and copper for robotic applications [15]. Electrode layers were formed by electroplating copper onto a PDMS layer. Five separate layers, including a bump layer, upper and lower electrode layers, an insulation layer and a spacer layer are bonded to form a sensing unit. An array of 256 sensing units on the same substrates results in a total sensor area of $22 \mathrm{~mm} \times 22 \mathrm{~mm}$. To sense the proximity event, two neighbouring electrodes form a capacitance based on the fringe effect of which the capacitance reduces when the object approaches; a maximum detection distance of $170 \mathrm{~mm}$ is achieved. Although the above research illustrates the principle of capacitive detection of human proximity, none of them have been realised on fabric with which their fabrication techniques are not compatible.

To enable a fabric-based proximity sensor, Wijesiriwardana et.al. reported a proximity sensor array made by knitting conductive polymer yarns into polyester fabric to form the electrodes [16]. An array $(3 \times 3)$ of nine electrodes with a rectangular pattern of $15 \mathrm{~mm} \times 18 \mathrm{~mm}$ was knitted. The electrode in the middle acts as the sensing electrode and the other eight 
surrounding electrodes are grounded. Due to the limitations of knitting, a dielectric fabric has to be attached separately on top of the knitted electrodes to avoid any unexpected electrical connection. Norgia et.al. reported a capacitive proximity sensor as a safety switch for cutting off the power from a chainsaw when it is too close to the operator [18]. The conductive wire cloth embedded within the whole garment acts as one electrode and the chainsaw is the other one. When the chainsaw is close to the conductive wire cloth, the safety switch is triggered and the power of the chainsaw is cut off. As a result, $100 \mathrm{~mm}$ was achieved as a safety distance for the power being cut off. No detailed results on the distance detected are given and the authors describe this approach as a near field proximity sensor. None of these reported proximity sensors are fabricated by printing directly on the fabric. They all require a complex fabrication process (e.g. MEMS microfabrication processes, knitting) with the limitations described earlier.

This paper reports dispenser printing of a proximity sensor on a $100 \%$ polyester woven fabric. The capacitive proximity sensor consists of a one-layer structure to detect proximity capacitively. This layer is a conductor layer which is the sensing electrode forming one electrode of the capacitor; the object to be detected forms the other electrode. Three electrode designs: filled, spiral and loop, are evaluated in this paper and compared to obtain a trade-off between the amount of ink used and the maximum detection distance. A simple detection circuit based on a proximity sensor chip is presented to allow ease of operation.

\section{Proximity sensing mechanisms}

Potential mechanisms that can be used to detect the presence of an object are: inductive [21], optical [19], ultrasonic [20] and capacitive [15]. The inductive mechanism generates an electromagnetic field and detects the eddy current losses when ferrous and nonferrous target objects enter the field [21]. Inductive proximity sensors normally consist of a metal coil, an oscillator, signal detector and an output circuit. The optical mechanism uses light reflection to determine the distance. Different light emitting sources can be used depending on the application, such as lasers with different wavelengths. The ultrasonic mechanism uses a similar principle to optical but based on an ultrasonic wave so colour and transparency of the object do not affect the results. The sensor radiates a series of short ultrasonic pulses and listens for their return from the reflecting object. Once the echo is detected, the distance is then acquired. The ultrasonic mechanism strongly depends on the transmission of air and the sonic reflectivity of the object. The capacitive sensing mechanism changes the capacitance due to the presence of an object. As the approaching object affects the electric field, the capacitance is changed.

Table 1: Comparison between common proximity sensing mechanisms.

\begin{tabular}{|c|c|c|c|c|c|}
\hline $\begin{array}{l}\text { Sensing } \\
\text { mechanism }\end{array}$ & $\begin{array}{l}\text { Sensing } \\
\text { element }\end{array}$ & $\begin{array}{l}\text { Detection } \\
\text { objects }\end{array}$ & $\begin{array}{l}\text { Normal } \\
\text { range }\end{array}$ & $\begin{array}{l}\text { Normal detection } \\
\text { circuit }\end{array}$ & Notes \\
\hline Inductive & A metal coil & Conductive & $\begin{array}{l}\text { Coil size } \\
\text { dependent }\end{array}$ & $\begin{array}{l}\text { LCR oscillator, } \\
\text { impedance } \\
\text { analyser }\end{array}$ & None \\
\hline Optical & $\begin{array}{l}\text { Lighting } \\
\text { source }\end{array}$ & $\begin{array}{l}\text { Conductive } \\
\text { and non- } \\
\text { conductive }\end{array}$ & $\begin{array}{l}\text { Frequency } \\
\text { and } \\
\text { condition } \\
\text { dependent }\end{array}$ & $\mathrm{I}-\mathrm{V}$ converter & $\begin{array}{l}\text { Lenses and } \\
\text { object } \\
\text { preparation } \\
\text { needed }\end{array}$ \\
\hline Ultrasonic & $\begin{array}{l}\text { Sound } \\
\text { generator }\end{array}$ & $\begin{array}{l}\text { Conductive } \\
\text { and non- } \\
\text { conductive }\end{array}$ & $\begin{array}{l}\text { Frequency } \\
\text { and } \\
\text { condition } \\
\text { dependent }\end{array}$ & $\begin{array}{l}\text { Sensor modules } \\
\text { or digital-to- } \\
\text { analog converter }\end{array}$ & $\begin{array}{l}\text { Object } \\
\text { dependent }\end{array}$ \\
\hline Capacitive & $\begin{array}{l}\text { A } \\
\text { conductive } \\
\text { electrode }\end{array}$ & $\begin{array}{l}\text { Conductive } \\
\text { and non- } \\
\text { conductive }\end{array}$ & $\begin{array}{l}\text { Conductive } \\
\text { electrode } \\
\text { size } \\
\text { dependent }\end{array}$ & $\begin{array}{l}\mathrm{RC} \text { low pass } \\
\text { filter, charge } \\
\text { amplifier, } \\
\text { capacitance meter }\end{array}$ & None \\
\hline
\end{tabular}


Table 1 summarises the key parameters of each proximity sensing mechanism. Inductive sensing can only detect conductive objects and the maximum detection range is about the same as the diameter of the sensing coil [21]. Even though both optical and ultrasonic mechanisms are able to detect conductive and non-conductive objects, the complex light source or sound generator respectively are difficult to achieve on fabric. In addition, the detection distance is dependent on the surface finish and material properties of the object being detected. The capacitive mechanism is able to detect both conductive and nonconductive objects so is suitable for detecting people and passive objects. The capacitive mechanism is also simple to set up and needs fewer components compared with other sensing mechanisms. It is the most suitable mechanism for a printed realisation since a simple conductive electrode of any shape can be used as the sensing element. A simple sensing element of any shape is advantageous for creative applications as the sensor can be designed to any artistic shape to meet the designer`s requirements.

\section{Design and fabrication}

\subsection{Proximity sensor design}

There are two potential sensor configurations [23], shown in Figure 1, of the capacitive proximity sensor.

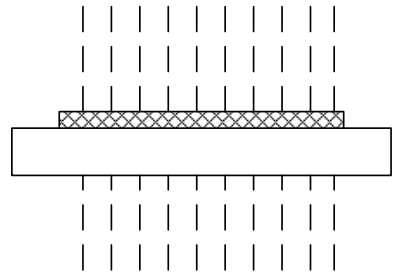

(a)

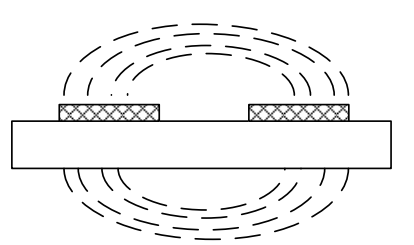

(b)

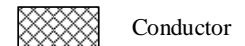

Substrate

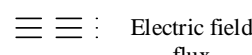

flux

Figure 1: Two configurations for a capacitively sensing proximity sensor: (a) single electrode and (b) two in-plane electrodes.

Configuration (a) is based on the principle of a parallel plate capacitor and only requires a single conductive plate to form one electrode of the capacitor. The object acts as a virtual earth. When an object, such as a human hand, approaches, the capacitance between the sensing electrode and the object increases. However, the capacitance can also be altered by other surrounding objects resulting in a false detection. Configuration (b) is based on the principle of electric field fringe effects and consists of two conductive electrodes adjacent to each other in the same plane. One acts as the positive electrode and the other acts as the negative electrode. Approaching objects influence the electric field between the two electrodes and the capacitance reduces when the object approaches. In this work, the single electrode configuration (a) is selected because it has a simpler structure and is easier to print compared with configuration (b) so is more suited to larger scale applications. In addition, since only a single conductive electrode is required, the geometric design of such electrode has no restrictions therefore suiting creative applications in which the appearance of the sensor may be important.

The influence of the sensing electrode design on the performance was evaluated by using three patterns with the same outer dimensions $(L \times L=40 \mathrm{~mm} \times 40 \mathrm{~mm})$, shown in Figure 2. 


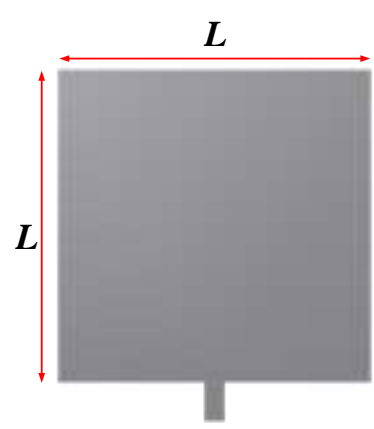

(a)

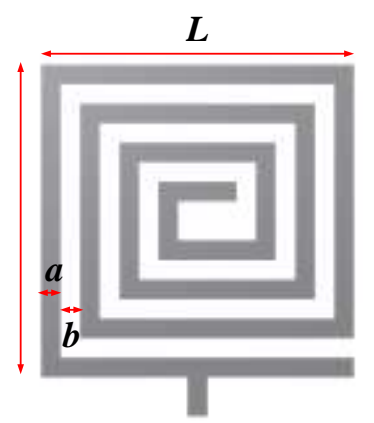

(b)

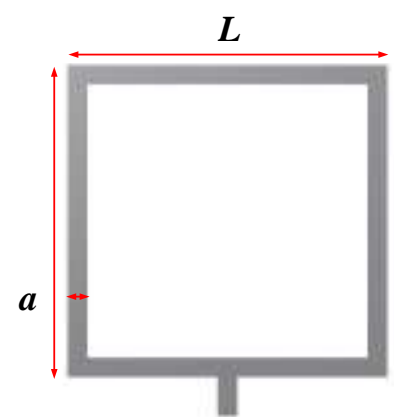

(c)

Figure 2: Three sensing electrode patterns (shown in grey) for the initial evaluation.

Electrode (a) is a standard filled pattern with the conductive material being evenly printed throughout the entire area. Electrode (b) is a spiral design in which both the width of the track $\boldsymbol{a}$ and the gap between tracks $\boldsymbol{b}$ is $2.5 \mathrm{~mm}$. Electrode (c) is a design in which the conductive material is only printed in a loop of which the track width $\boldsymbol{a}$ is $2.5 \mathrm{~mm}$. In this particular example, square patterns are printed to suit the design although other geometric patterns are possible such as circular.

\subsection{Printing of the capacitive proximity sensor}

The dispenser printer is a bespoke machine [3-5] developed at the University of Southampton. The material is deposited via a pressurised syringe onto the substrate which is controlled in three dimensions using an XYZ stage system according to the desired printed pattern. The printing resolution is dependent on the accuracy of each moving stage, the size of the syringe nozzle and the material rheology. The amount of ink deposited is controlled by the pressure acting on the syringe and the printing mode used (i.e. droplet or continuous printing).

The substrate used is a $360 \mu \mathrm{m}$ thick, $100 \%$ polyester woven fabric supplied by Berger Textil, Germany. This fabric is one of the most common fabrics used in the creative industries and is the base material for inkjet printed display banners, exhibition stands and window blinds [24]. A woven fabric was chosen for this work because it is more common in the construction of these applications than plastic film; fabric will conform to the desired structure more easily and the weave structure allows the fabric to stretch where needed without damaging the printed layers. Fabinks TC-C4001, supplied by Smart Fabric Inks ltd [25], is used as the conductor since it is compatible with dispenser printing and the fabric. Once cured at $130{ }^{\circ} \mathrm{C}$, which does not damage the fabric, it has a conductivity of $1.2 \times 10^{6} \mathrm{~S} / \mathrm{m}$. Before printing, the fabric is pre-cut to $100 \mathrm{~mm}$ by $100 \mathrm{~mm}$ and then mounted onto an alumina tile as a rigid supporting platform. The dispenser printing parameters are summarised in Table 2. The conductive ink consists of silver flakes blended with a polymer binder, printing enhancement additives and solvent. The printed films pass the standard industrial tape and scratch tests but full abrasion resistance has not been tested.

Table 2: Printing parameters of the conductive ink.

\begin{tabular}{ll}
\hline Parameter & Value \\
\hline Dispensing time & $30 \mathrm{~ms}$ \\
Dispensing pressure & $50 \mathrm{kPa}$ \\
Vacuum pressure & $1 \mathrm{kPa}$ \\
x-resolution & $0.5 \mathrm{~mm}$ \\
y-resolution & $0.4 \mathrm{~mm}$ \\
Curing & $130^{\circ} \mathrm{C} 15$ mins \\
\hline
\end{tabular}




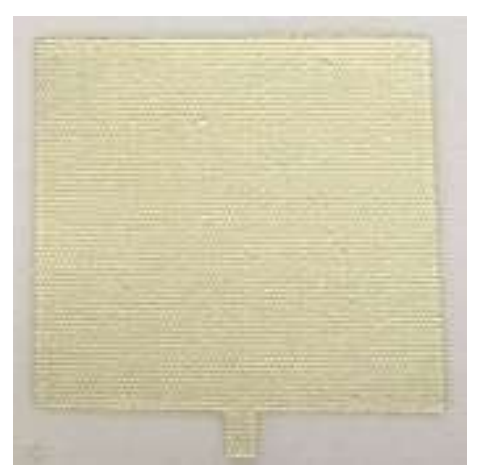

(a)

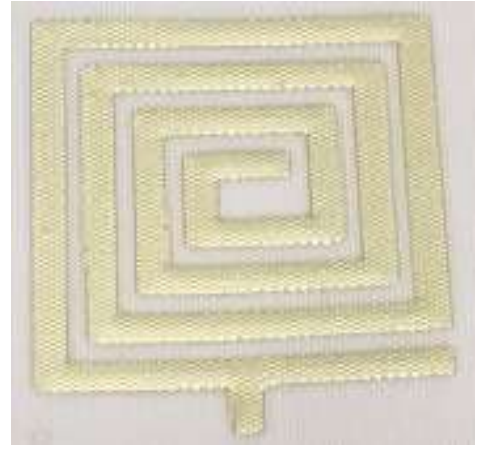

(b)

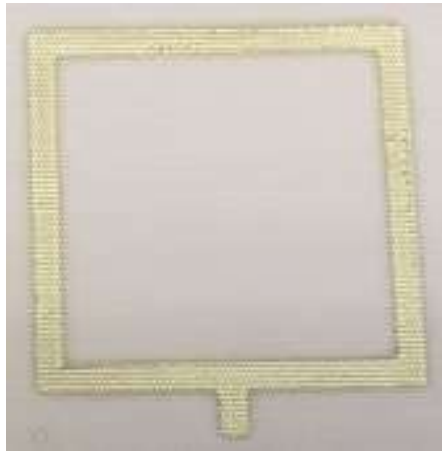

(c)

Figure 3: Printed proximity sensors on fabric with different designs: (a) filled, (b) spiral and (c) loop.

Figure 3 shows three printed proximity sensor designs on the fabric. The printed conductor surface is smooth without any bubbles or wrinkles and an average thickness of $35 \mu \mathrm{m}$ over three designs is obtained. There is no observable damage to the fabric due to the curing process, confirming compatibility between the ink and fabric.

\section{Proximity sensor testing}

\subsection{Sensing circuit design}

All sensing circuits reported in the literature are bespoke and no detailed information on their detection method is disclosed. To achieve the proximity sensor functionality, a commercially available proximity sensor integrated circuit (IC), Microchip MTCH112, is selected [26]. The MTCH112 has a maximum supported input capacitance of $40 \mathrm{pF}$ and can be configured to either provide two independent capacitive proximity sensing channels or to provide one sensor channel and an active guard electrode which reduces susceptibility to external electrical noise. This can be used to reduce the effect of stray capacitance on the sensor electrode and the interconnections between the sensor electrode and the IC. It automatically adjusts, and is able to reduce, the effect of environmental effects whilst still allowing transient effects to be observed. In addition, the MTCH1 12 does not require any external components to operate and has a small footprint of $3 \mathrm{~mm}$ by $3 \mathrm{~mm}$, therefore providing a space efficient solution.

The MTCH112 produces a 13 bit value which represents the proximity sensor reading; it also produces an automatically tracking baseline level reading, as a 13 bit value, which provides a reference related to environmental conditions. By considering the difference between the sensor reading and the baseline value, the change in sensor reading due to the introduction of an object can be distinguished from the sensor reading attributable to the sensor interconnections and stray capacitances due to the mounting of the smart textile.

For this particular application, the printed proximity sensor is connected to one sensing channel through a coaxial cable of which the metallic shield is connected to the other sensing channel to achieve an active guard. Using a coaxial cable will minimise the influence from the surrounding objects during the testing and this cable is only connected to the edge of the creative application thus not interfering with the aesthetic of the design. The entire circuit is driven by a PC through a USB cable and the proximity detection event is indicated by an LED which is connected at the output pin of the MTCH 112.

\subsection{Comparison of the proximity sensor designs}

The three designs shown in Figure 3 were first simulated using COMSOL Multiphysics finite element modelling using the Electrostatics module to evaluate the capacitance change as a function of distance. In the simulation, the distance represents that between the sensing electrode, shown in Figure 3, which is of thickness $30 \mu \mathrm{m}$ and a second conductive plate of dimensions $18 \mathrm{~cm}$ by $12 \mathrm{~cm}$ by $2 \mathrm{~cm}$ which represents the human hand. The separation 
between the two plates is air and the mesh was defined as 'extremely fine' for the two plates and the air in between. The material parameters (conductivity, dielectric constant and density) of the printed silver sensing plate and the fabric were approximated by those of pure solid silver and polyester.

In the simulation, the driven voltage is $3.3 \mathrm{~V}$ which is the maximum operating voltage of the proximity sensing IC. Figure 4 shows the simulated capacitance change as a function of distance.

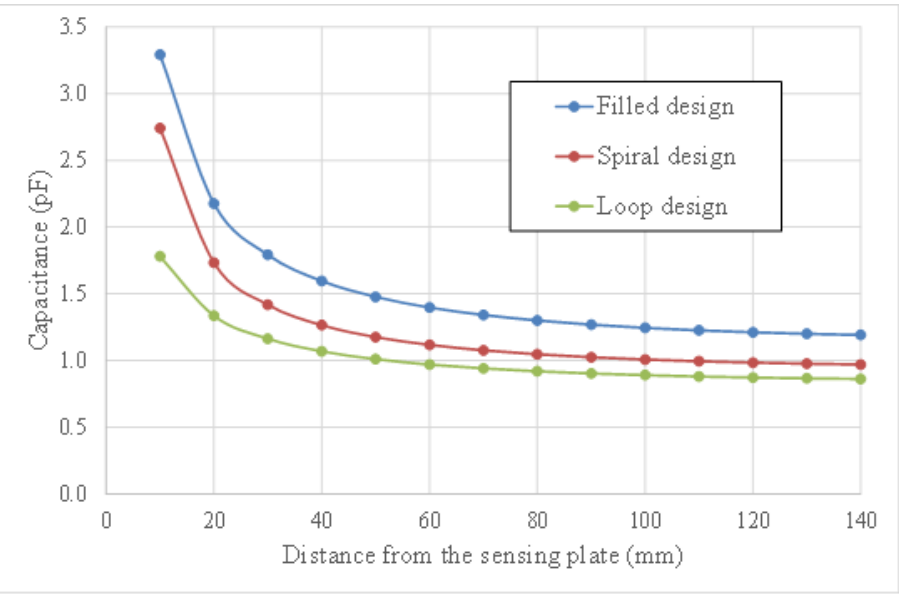

Figure 4: COMSOL capacitance change simulation.

The simulated capacitance change of the loop design is smaller than that of the other two designs for all simulated distances because of its smaller electrode area whereas the filled design has the maximum capacitance change of the three designs. Each proximity sensor was individually connected to the sensing circuit and was laid on a horizontal flat surface to minimise the stray capacitance and maximise the percentage change in capacitance. A human hand vertically approached the sensor from above as this approach produces the maximum change in capacitance resulting in a reference performance. If the sensor plate is approached at an angle to the vertical then the sensor output will be reduced. The detection distance was measured using a plastic ruler, to avoid potential interference, which was located next to the sensor. Three samples of each design were tested three times under the same conditions. Figure 5 compares the maximum detection distance of the three proximity sensor designs. The simulation in Figure 4 shows that the overall trend is that, after approximately $80 \mathrm{~mm}$, the change in capacitance is small which ultimately limits the maximum detection distance.

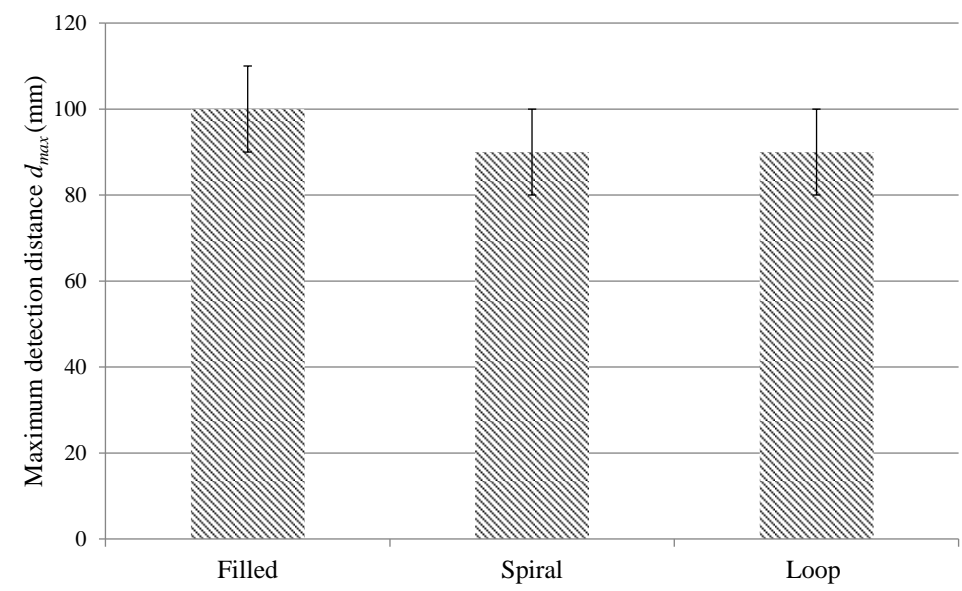

Figure 5: Maximum detection distances of three designs. 
The filled pattern has a slightly higher detection distance as its capacitance change is the highest of the three designs. The maximum detection distance of the filled pattern is $10 \%$ higher than that of the other two designs due to the size of the conductive area which matches with the results shown in Figure 4. The amount of conductive ink used to print the three designs was $0.96 \mathrm{~g}, 0.54 \mathrm{~g}$ and $0.23 \mathrm{~g}$ for the filled, spiral and loop designs, respectively. There is a less than $1 \%$ difference between the loop and spiral designs in terms of the maximum detection distance. The loop design is therefore the preferred solution offering a compromise between detection range and quantity of ink required: it uses $\sim 76 \%$ less ink but still offers $90 \%$ of the range of the filled design.

To quantify the performance of the proximity sensing for a given conductive area, the detection coefficient $\left(\eta_{d}\right)$, defined as the ratio of the maximum detection distance $\left(d_{\max }\right)$ to the total conductive area $\left(A_{c s}\right)$ used as the sensing electrode, is introduced.

$$
\eta_{d}=\frac{d_{\max }}{A_{c s}}
$$

For each sensing electrode design, the detection coefficient is shown in Figure 6.

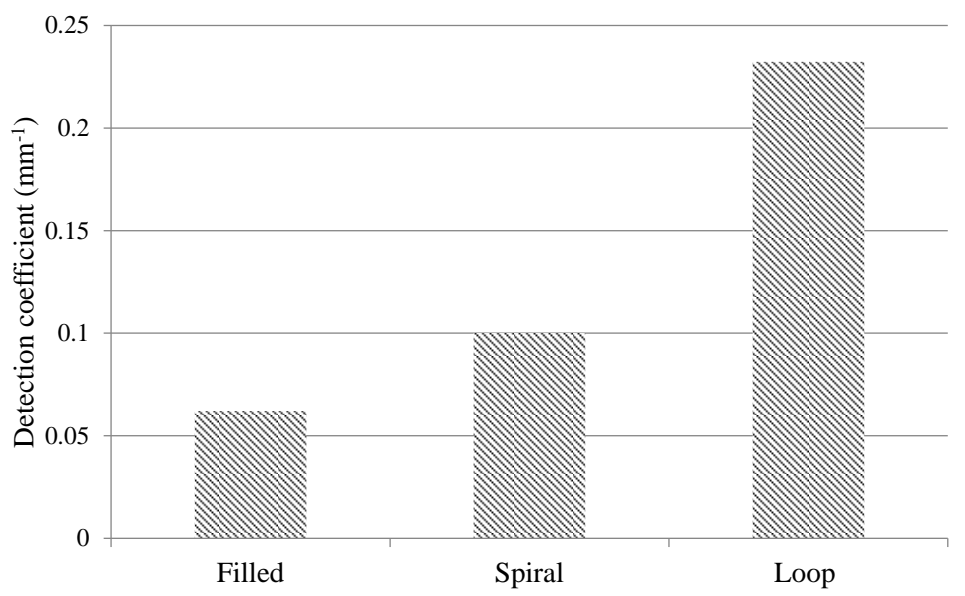

Figure 6: Detection coefficient of each sensing electrode design.

It is shown that the loop design achieves the maximum detection coefficient (i.e. 0.23) of the three designs. Considering the amount of ink being used for the filled and spiral designs, the loop design requires less conductive ink and reduces the area of the fabric being printed so that the printing time required to produce a large area can be minimised and the impact on the fundamental fabric properties, such as breathability and flexibility, are minimised.

\subsection{Comparison of loop designs with different width ratios}

The ratio of the track width $\left(L_{1}\right)$ to the total width of the proximity sensor electrode $(L)$ is defined as $\sigma$, shown in Figure 7:

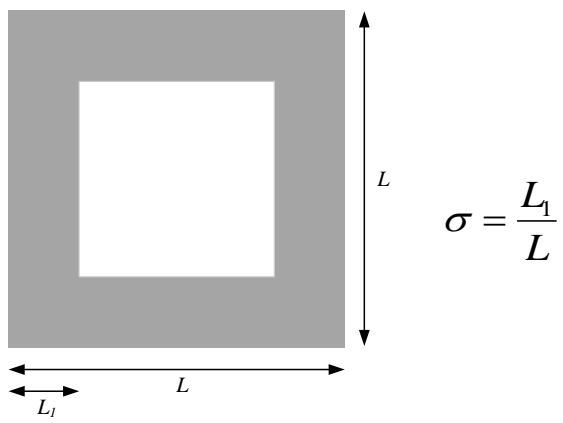


Figure 7: Width ratio $(\sigma)$ of the loop design.

In the previous section, a loop design with a width ratio of 1/16 was tested resulting in a loop design with the highest detection coefficient and least usage of conductive ink. To further investigate the loop design, the overall area was fixed at $40 \mathrm{~mm}$ x $40 \mathrm{~mm}$ and the width of the loop $L_{l}$ varied, as shown in Figure 8.

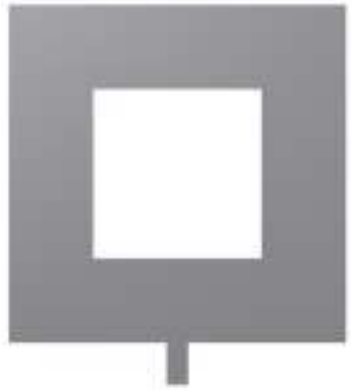

(a)

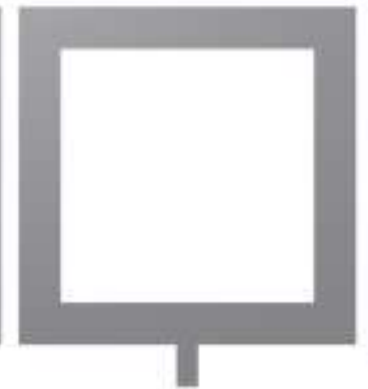

(b)

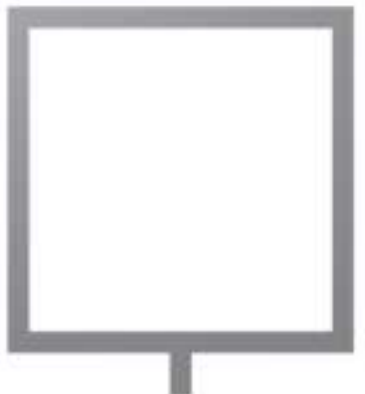

(c)

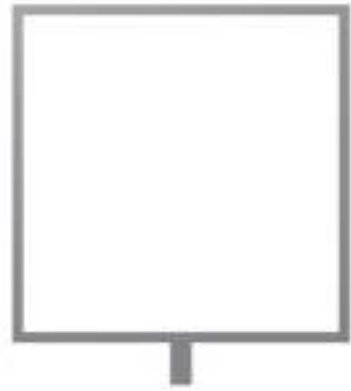

(d)

Figure 8: Loop designs with the width ratio of: (a) $\sigma=1 / 4$; (b) $\sigma=1 / 8$; (c) $\sigma=1 / 16$; (d) $\sigma=1 / 32$.

The resultant printed proximity sensors are shown in Figure 9.

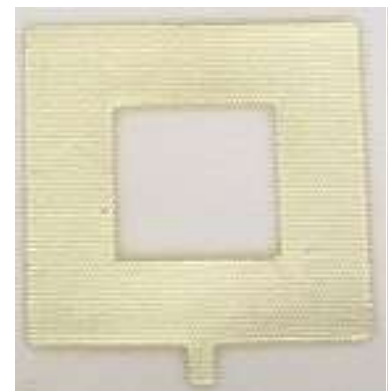

(a) (b)

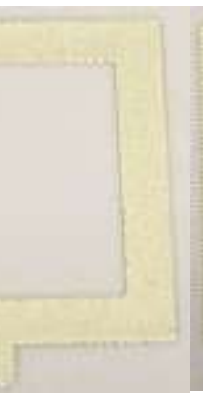

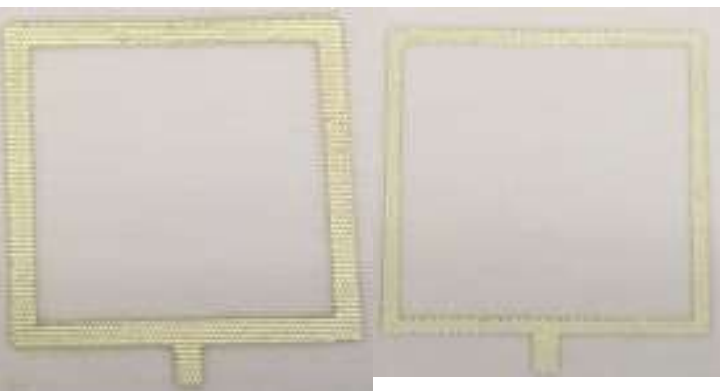

(c) (d)

Figure 9: Dispenser printed proximity sensors with the width ratios shown in Figure 8.

The amounts of conductive ink used to print the above designs are $0.72 \mathrm{~g}, 0.42 \mathrm{~g}, 0.23 \mathrm{~g}$ and $0.12 \mathrm{~g}$ for width ratios of $1 / 4,1 / 8,1 / 16$ and $1 / 32$, respectively. The maximum detection distance of each printed sensor was evaluated using the same setup with the sensor laid on a flat wooden lab bench. The results are shown in Figure 10.

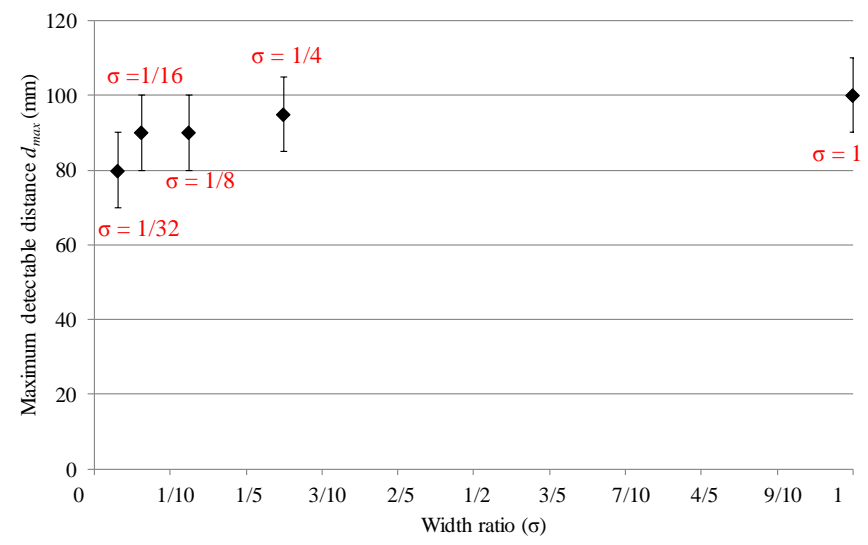

Figure 10: Maximum detection distance $\left(d_{\max }\right)$ as a function of the width ratio $(\sigma)$.

The detection coefficients are $0.06 \mathrm{~mm}^{-1}, 0.08 \mathrm{~mm}^{-1}, 0.12 \mathrm{~mm}^{-1}, 0.24 \mathrm{~mm}^{-1}$ and $0.41 \mathrm{~mm}^{-1}$ for each width ratio of $1,1 / 4,1 / 8,1 / 16$ and $1 / 32$, respectively. The results show that a smaller 
width ratio improves the detection coefficient but a width ratio of $1 / 32$ on fabric is the limit of the current printer. From Figure 10, it is found that the maximum detection distance does not change significantly when the ratio $(\sigma)$ is above $1 / 16$, but drops once the ratio is below this. There is no significant difference in terms of the maximum detection distance between the width ratios of $1 / 16,1 / 8$ and $1 / 4$ as the discrepancy between them is $\sim 5 \%$. Therefore, a ratio of $1 / 16$ is chosen as a trade-off ratio for the future sensor electrode design because:

- $1 / 16$ width ratio allows the proximity sensor to achieve $90 \%$ of the detection range of the filled sensor with a $1 / 1$ width ratio;

- $1 / 16$ width ratio uses $76 \%$ less conductive ink compared with that for the filled sensor and therefore minimises the amount of the conductor used to achieve a comparable detection distance

\subsection{Maximum detection distance as a function of sensing electrode size at a width ratio of $1 / 16$}

Having selected a width ratio of $1 / 16$ for the printed loop sensor, different outer lengths $(L)$, ranging from $10 \mathrm{~mm}$ to $400 \mathrm{~mm}$, were tested using the same setup and circuit described above. Figure 11 shows the maximum detection distance of different sized printed loop sensors with the same width ratio of $1 / 16$.

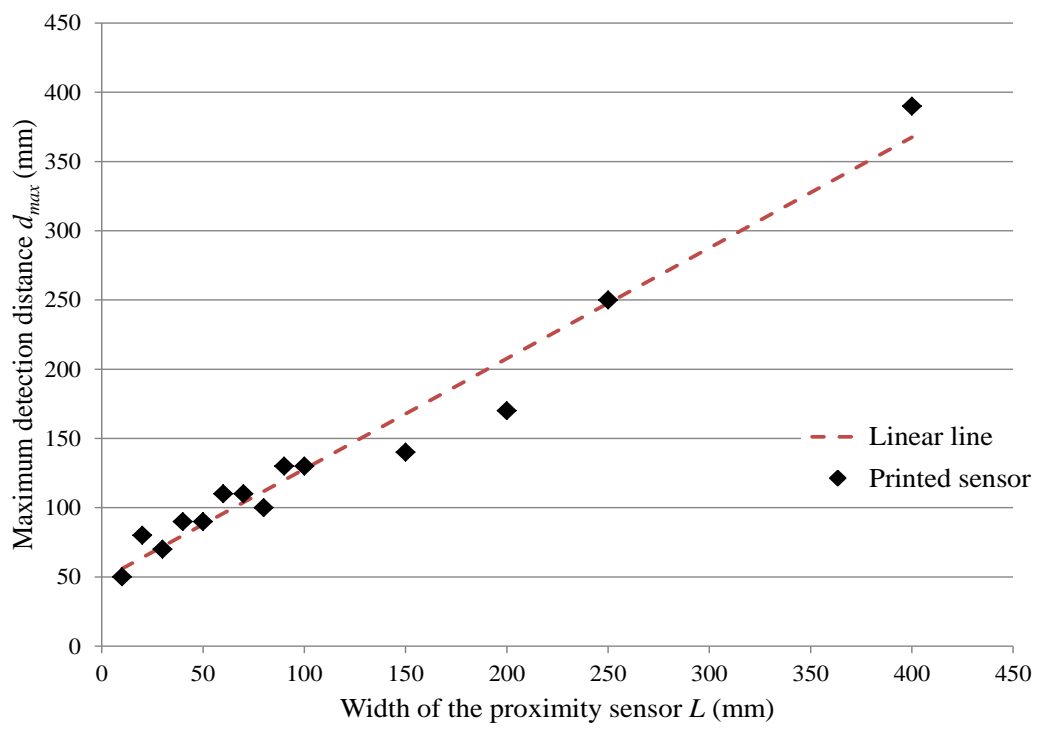

Figure 11: Maximum detection distance $d_{\max }$ as a function of the proximity sensor width $L$.

From Figure 11, it is shown that the maximum detection distance increases with the sensor size. The linearity of the sensing circuit is then calculated resulting in 0.79 and therefore allowing the hypothesis that any electrode larger than $400 \mathrm{~mm}$ will produce a maximum detection distance being approximately equal to its width.

\section{Conclusions}

A capacitive proximity sensor has been dispenser printed onto a polyester fabric in the form of a loop design. The loop design is selected because the quantity of conductive ink is reduced by $76 \%$ whilst achieving $90 \%$ of the maximum detection distance compared with the standard filled design. The loop design also has the highest detection coefficient, comparing detection distance with conductive area, of 0.23 compared with 0.06 for the filled and 0.1 for the spiral designs. A width ratio of $1 / 16$ is selected because it is the lowest ratio at which the maximum detection distance is not significantly $(\sim 10 \%)$ lower compared with the filled design. In addition, using the loop design maximises the remaining fabric area for printing colour images or other functionality as the proximity sensor design can be placed around the border. 
Using a Microchip MTCH112 allows the simplicity of the sensing circuit to be achieved since no external components are needed for operation, thereby providing a space efficient solution and reducing the complexity of operation. Having tested printed proximity sensors with different sizes ranging from $10 \mathrm{~mm}$ to $400 \mathrm{~mm}$, the linearity of the sensing circuit is calculated to be 0.79 . A large detection distance implies that the proximity sensor is able to interact with the people in large-scale creative applications. In addition, the sensing circuit is based on a commercially available proximity sensor IC thus making this system potentially more compact and easier to obtain than the bespoke systems used in the literature.

\section{Acknowledgements}

The authors acknowledge the funding from the EU (Grant No.: CP-FP-INFSO-FP7-610414) for the smart fabrics research project CREATIF (http://www.creatif.ecs.soton.ac.uk) under which this work was carried out. The authors would like to thank Smart Fabric Inks Ltd for supplying the printable conductive paste.

All data supporting this study are openly available from the University of Southampton repository at http://doi:10.5258/SOTON/388277.

\section{References}

[1]. X. Tao, Wearable Electronic and Photonics, Woodhead Publishing and CRC press, 2005.

[2]. J. Meyer, P. Lukowicz and G. Troster, "Textile pressure sensor for muscle activity and motion detection," in 10th IEEE International Symposium on Wearable Computers, Montreux, Switzerland, 2006.

[3]. M. de Vos, R. Torah and J. Tudor, "A novel pneumatic dispenser fabrication technique for digitally printing electroluminescent lamps on fabric," in Symposium on Design Test Integration and Packaging of MEMS and MOEMS (DTIP), Montpellier, 2015.

[4]. Z. Ahmed, R. Torah and J. Tudor, "Optimisation of a novel direct-write dispenser printer technique for improving printed smart fabric device performance," in Symposium on Design Test Integration and Packaging of MEMS and MOEMS (DTIP), Montpellier, 2015.

[5]. Y. Wei, R. Torah, Y. Li and J. Tudor, "Dispenser printed proximity sensor on fabric for creative smart fabric applications," in Symposium on Design Test Integration and Packaging of MEMS and MOEMS (MEMS), Montpellier, 2015.

[6]. C. C. Ho, "Dispenser printed zinc microbattery with an ionic liquid gel electrolyte," Journal of Micromechanics and microengineering, 20, 2010.

[7]. R. Dorey, Ceramic thick films for MEMS and microdevices, Elsevier Inc. 2011.

[8]. S. Hoath, G. Martin and I. Hutchings, "Effects of fluid viscosity on drop-on-demand ink-jet break-off," in International Conference on Digital Printing Technology, 2010.

[9]. Optomec Aerosol System, Available at: http://www.optomec.com/printedelectronics/aerosol-jet-technology [Accessed: 19/2/2016].

[10]. E. Cheung and V. J. Lumelsky, "Proximity sensing in robot manipulator motion planning: system and implementation issues," IEEE Transactions on Robotics and Automation, 5, pp. 740-751, 1989.

[11]. R. Volpe and R. Ivlev, "A survey and experimental evaluation of proximity sensors for space robots," in IEEE International Conference on Robotics and Automation, 1994.

[12]. L. C. Maier, "Apparatus and method for determining liquid levels". US Patent 4908783 , 1987.

[13]. J. L. Novak and J. T. Feddema, "A capacitance-based proximity sensor for whole arm obstacles avoidance," in International Conference on Robotic and Automation, Nice, France, 1992.

[14]. T. Togura, K. Sakiyama, Y. Nakamura and K. Akashi, "Long range human body sensing modules with capacitive sensor," Automotive products R \& D department, Electronic Components R \& D, 2009. 
[15]. H. Lee, S. Chang and E. Yoon, "Dual mode capacitive proximity sensor for robot applications: implementaion of tactile and proximity sensing capacbility on a single polymer platform using shared electrodes," IEEE Sensors Journal, 9, pp. 1748-55, 2009.

[16]. R. Wijesiriwardana, K. Mitcham, W. Hurley and T. Dias, "Capacitive fiber-meshed transducers for touch and proximity sensing application," IEEE Sensors Journal, 5, pp. 989-94, 2005.

[17]. D. K. Lambert, "Capacitive proximity sensor". US Patent 6724324B1, 2004.

[18]. M. Norgia and C. Svelto, "RF-Capacitive proximity sensor for safety applications," in Instrumentation and Measurement Technology Conference, Warsaw, Poland, 2007.

[19]. L. Burgi, R. Pfeiffer, M. Mucklich, P. Metzler, M. Kiy and C. Winnewisser, "Optical proximity and touch sensors based on monolithically integrated polymer photodiodes and polymer LEDs," Organic Electronics, 7, pp. 114-20, 2006.

[20]. S. Min, J. Kim, H. Shin, Y. Yun, C. Lee and M. Lee, "Noncontact respiration rate measurement system using an ultrasonic proximity sensor," IEEE Sensors Journal, 10, pp. 1732-39, 2010.

[21]. S. Fericean and R. Droxler, "New noncontacting inductive analog proximity and inductive linear displacement sensors for industrial automation," IEEE Sensors Journal, 7, pp. 1538-45, 2007.

[22]. L. K. Baxter, Capacitive sensors: Design and Applications, New York: Institute of Electrical and Electronics Engineers, Inc., 1997.

[23]. B. Osoinah, "Proximity capacitive sensor technology for touch sensing applications," Freescale semiconductor Inc. Proximity sensing white paper.

[24]. Berger Textil datasheet, 'Back-Lighttex Fr $+\mathrm{W}$ ', Available at: http://www.mediaoneusa.com/assets/images/DataSheet/4499-6-180.pdf [Accessed: $22 / 02 / 2016]$.

[25]. Smart fabric inks, Available at: http://www.fabinks.com. [Accessed: 22/02/2016].

[26]. Microchip datasheet, " MTCH112 Dual-channel proximity/touch controller ". Available at: http://ww1.microchip.com/downloads/en/DeviceDoc/41668A.pdf [Accessed: 22/02/2016]. 